\title{
Uniqueness and Reproducibility of Semantic Intelligence: New Approach to the Notion of Self-Organization
}

\author{
Maria K. Koleva \\ Institute of Catalysis, Bulgarian Academy of Sciences, Sofia, Bulgaria \\ Email: mkoleva_1113@yahoo.com
}

How to cite this paper: Koleva, M.K. (2019) Uniqueness and Reproducibility of Semantic Intelligence: New Approach to the Notion of Self-Organization. Journal of Modern Physics, 10, 43-58.

https://doi.org/10.4236/jmp.2019.101005

Received: January 3, 2019

Accepted: January 20, 2019

Published: January 23, 2019

Copyright (อ 2019 by author(s) and Scientific Research Publishing Inc. This work is licensed under the Creative Commons Attribution International License (CC BY 4.0).

http://creativecommons.org/licenses/by/4.0/

\section{(c) (i) Open Access}

\begin{abstract}
A novel notion of self-organization whose major property is that it brings about the execution of semantic intelligence as spontaneous physico-chemical processes in an unspecified ever-changing non-uniform environment is introduced. Its greatest advantage is that the covariance of causality encapsulated in any piece of semantic intelligence is provided with a great diversity of its individuality viewed as the properties of the current response and its reproducibility viewed as causality encapsulated in any of the homeostatic patterns. Alongside, the consistency of the functional metrics, which is always Euclidean, with any metrics of the space-time renders the proposed notion of self-organization ubiquitously available.
\end{abstract}

\section{Keywords}

Self-Organization, Concept of Boundedness, Point-Like Approach to Response, Semantic Intelligence, Algorithmic Intelligence, Metrics

\section{Introduction}

So far the dominant concept of the interdisciplinary science is that the hierarchy of complexity of natural systems develops as decentralized form of self-organization which is going bottom up. This short statement implies that since each and every complex system is comprised by atoms and molecules, its emergent behavior can be derived as a decentralized self-organization of constituting atoms and molecules subject to simple rules. Despite the promising results about the development of pattern formation and swarm intelligence, this idea suffers serious weakness in a number of key points and needs serious modification. Thus, the grounding idea of swarm intelligence, namely the emergence of collective beha- 
vior out of decentralized simple rules, is inherently contradictive since a successful self-organization involves a rule called cohesion whose implementation needs permanently information about the current center of mass of the corresponding system. This, however, implies that the rule cohesion is not a simple dynamical rule but it is also an emergent on higher levels of complexity rule typical for a rather mindful behavior. The inherent contradiction of pattern formation scenario will be considered in Section 3.

Another weak point of the above idea is that the emergent from simple rules behavior cannot explain the autonomous way in which the natural semantics is accomplished. This is a result of the fact that emergent behavior commencing from the traditional setting of self-organization is accomplished as a short transit to a steady asymptotic. Thus, neither form of intelligence can proceed autonomously in the frame of such view on self-organization.

There are other weak points of the above idea of self-organization some of which will be discussed later in the paper and more of them can be found in the author's recent book [1]. The major conclusion drawn from those weaknesses is that the idea of self-organization needs fundamental revision. I find that the problem so serious that its resolution requires a fundamentally novel view on it. The revision started with the introduction of the idea that there exists general operational protocol supervising dynamical behavior of stable natural systems which I called boundedness. Putting in a nutshell, it means that a stable system develops so that both amplitudes and rates of exchange of matter, energy and information with its environment are kept permanently bounded within specific to its margins. A systematic development of that idea is presented in [1]. The theory successfully resolves the major difficulties which the behavior of complex systems challenges the present day interdisciplinary science. However, the major advantage of that theory is the substantiation of a new type of intelligence, called by me semantic intelligence, whose major property is autonomous creation and comprehension of information in an ever-changing non-uniform environment. The new type of intelligence is viewed as an emergent dynamical phenomenon where the autonomity of semantics is implemented by means of spontaneous motion among different steady patterns each of which stands for a semantic unit. The novel, and to the much extent surprising, grounds for the existence of steady spatio-temporal patterns in a non-uniform ever-changing environment is the central for the entire theory of boundedness proof of the called by me decomposition theorem. The latter asserts that for each and every bounded irregular sequence (BIS) there exists presentation basis (which turns to be power spectrum) where the spectrum additively decomposes into two parts: a specific steady spatio-temporal patter pattern, called homeostasis, and a universal "noise" component so that both the details of the pattern and the shape of the noise component are robust to details of the statistics of the BIS.

There are several major outcomes of the decomposition theorem:

1) The first one is that this decomposition makes possible to distinguish the causal relations encapsulated in the homeostatic patterns from the non-causal 
ones encapsulated in the noise term. Thus, the response viewed as a bounded irregular sequence of current response is unique while its decomposition in the corresponding power spectrum provides the reproducibility of the corresponding semantics viewed as a system of steady causal relations encapsulated in the corresponding homeostatic pattern.

2) Since each and every homeostatic pattern is an extended in space and time object, it poses the question about the character of the dynamical rules that produce that behavior.

The importance of the latter problem stems from the controversy with another basic idea of the traditional science: that of point-like approach to response. In a nutshell, the latter implies that in equilibrium, the response of a system or any of its sub-unit to a uniform steady environment is applied to a single spatial point called center of mass and remains the same forever if not other forces are applied. Thus, the formation of a response is set up by a single spatio-temporal point. This requirement commences from the mechanical view that a system in equilibrium does not rotate. The steady in the time behavior commences from the Newton's third law. The above considerations make clear where the name point-like response comes for.

These basic mechanical views are extended to systems of different nature and different levels of complexity through generalizing the idea of mechanical equilibrium to the idea of thermodynamic equilibrium viewed as a general Law of Nature. This idea consists of assertion that to be in thermodynamic equilibrium, the response of each and every system to any environmental impact proceeds as the development of the corresponding spontaneous fluctuation. Further, it is taken for granted that the process of development of any fluctuation in the course of time is irrelevant, because it is taken for granted that the thermodynamic equilibrium is a global attractor for all initial states. In turn, this suggests that any deviation from the thermodynamic equilibrium is due to damp out in a finite time in a spontaneous way. This assumption serves as grounds for adoption the idea that all fluctuations (and accordingly the responses to environmental impacts) can be presented as instantaneous independent events subject to probability distribution and so they become subjects of the Central Limit Theorem. As a result, this generalization culminates in the validation of the Central Limit Theorem as the most widespread tool for studying spatio-temporal phenomena of different origin.

Little or no attention has been paid to the fact that this generalization holds only for steady uniform environment. It is also taken for granted that: 1) all fluctuations damp out spontaneously and irrespectively to their amplitude; 2) no constraints on the amplitude of fluctuations (and correspondingly to the environmental impact) are imposed.

The major and non-overwhelming difficulty of that generalization is the fact that the subject of the Central Limit Theorem is generically irreconcilable with any form of intelligence, since the latter always is viewed as a sequence of de- 
pendent from one another event while the Central Limit Theorem holds only for independent events.

Now it becomes evident that the decomposition theorem is more adequate frame for any form of intelligence. On the other hand, however, it's subject is completely different from the subject of the Central Limit Theorem. Indeed, while the subject of the decomposition theorem are bounded irregular variables (yet not independent), the subject of the Central Limit are independent variables (yet not bounded). It should be stressed on the fact that the subjects of both theorems have not cross-section because any form of permanent boundedness implies long-term correlations.

Outlining, the decomposition theorem sets the general frame for substantiation of the long-term correlations among semantic units. Now we face the major problem how to modify the idea about the point-like approach to response and the idea of self-organization so that to match physically the proceeding of semantic intelligence in the autonomous way. It will be demonstrated that an appropriate modification of certain basic notions and ideas of physics and certain modification of the idea of self-organization, provides the targeted physical implementation of the semantic intelligence. It will be seen that this issue is crucially related to the problem about uniqueness and reproducibility of the semantic intelligence.

\section{Point-Like Approach to Response: Critical Overview}

The goal of the present section is to provide a critical analysis of all pro and cons of one of the grounding ideas of the nowadays interdisciplinary science, namely the idea of point-like approach to response. In a nutshell the notion of point-like approach to response has two-fold meaning:

1) To be in a mechanical equilibrium it is necessary that each and every single object does not rotate. This is possible if only all mechanical forces are applied in a single point called center of mass and all forces acts at the same time. The next important point is that the location of the center of mass does not change its position within the corresponding object. The greatest advantage of that idea is that it provides time translational invariance of the physical laws that governs the dynamics of any object as well as its spatial covariance, i.e. the independence of those laws from the reference frame chosen for description of the corresponding motion.

2) To be in thermodynamic equilibrium, it is supposed that the response to any environmental impact matches the development of the corresponding spontaneous fluctuation. This is the assertion of the so call fluctuation-dissipation theorem. Since the notion of thermodynamic equilibrium implies that it is a global attractor for all initial conditions, it is taken for granted that the development of any fluctuation in the course of time and in space is irrelevant because the fluctuations are supposed to develop coherently throughout a system. This assumption serves as grounds for adoption the idea that all fluctuations (and accordingly the 
responses to environmental impacts) can be presented as instantaneous independent events subject to a probability distribution and so they become subjects of the Central Limit Theorem. It is worth noting that what is tacitly implied about these assumptions is that the environment is supposed uniform and steady; fluctuations damp out spontaneously and irrespectively to their amplitude; no constraints on the amplitude of fluctuations (and correspondingly to the environmental impact) are imposed.

The idea of mechanical equilibrium is developed further into the idea that the environment of any object provides a specific time-independent Hamiltonian which turns out to be the bearer of identity of the corresponding object. The admissible states of the object are defined by means of imposing specific initial and boundary conditions to the dynamical rule which comprises the corresponding Hamiltonian. This setting commences from the idea that there exists presentation basis such that all dynamical interactions are considerable as isolated from one another events. In turn the latter renders spatial and temporal dimension of any isolated interaction irrelevant for the outcome of its processing, hence the name of point-like approach to response.

Further, to this general protocol, the assumption that steady motion is available only on those trajectories where the action is stationary is added. Thus, the latter idea selects that single trajectory which provides stationarity of the corresponding action. It should be stressed that so far the stationarity of the action is always derived from the constraint of some extermination such as being the shortest path, being the trajectory where the energy loses are minimal, the trajectory where the entropy is maximal and so on. A persistently overlooking fact is that this renders the succession of states which a trajectory, subject to stationary action, passes through mutually dependent.

However the latter result is apparently incompatible with the Central Limit Theorem. Indeed, the latter is grounded on the fluctuation-dissipation theorem viewed as assertion that the fluctuations and correspondingly all responses are independent from one another events and thus each of them can precede and/or succeed any other one. Put it in more formal terms it asserts that any succession of fluctuations/responses forms a Markov process. To remind that indeed the notion of a Markov process implies that a succession of jumps has memory of bounded radius. Thus, all events, or blobs of events, are to be considered as independent from one another events and thus each and every Markov process is subject to the Central Limit Theorem. The idea of Markoviniaty implies that all trajectories are admissible which, however, is incompatible with the idea about stationarity of the action viewed as the general protocol which selects the sub-set of admissible trajectories from the set of all available. So far the dominant concept is that of the Markovianity. It, however, is not able to substantiate any form of semantic and/or algorithmic intelligence, because the fundamental difference between any piece of semantics and/or algorithm on the one hand, and a random sequence on the other hand, implies inter-dependence among the succession of the constituting events in the former case and its lack in the case of ran- 
dom sequences.

The greatest value of the point-like approach is that it provides time-translational invariance of the laws of Nature and their independence from the reference frame chosen for their description. However, the revealed in the Introduction and in the present section inherent contradictions and incapability for explaining the uniqueness and reproducibility of any semantics, viewed as a specific sequence of mutually dependent events, call for necessity of a fundamental revision. A successful revision should provide not only the time-translational invariance of the laws of Nature and their independence from a reference frame, but altogether it should provide a consistent with them explanation of the uniqueness and reproducibility of semantics.

\section{Self-Organization: Critical Analysis in a Nutshell}

Formal definition of semantic intelligence as well as its major properties is provided in Chapter 10 of [1]. There the semantic intelligence is viewed as a new form of intelligence whose major property is autonomous creation and comprehension of information. The latter property provides the demarcation line with the algorithmic intelligence which serves as ground for all types of modern-day computers and artificial intelligence. The major property of algorithmic intelligence is that its execution is possible only in artificially designed and artificially maintained environment. The main reason why the algorithmic intelligence cannot be executed by spontaneous processes in the frame of the point-like approach to response is that the very idea of execution of any form of intelligence is incompatible with the idea of thermodynamic equilibrium viewed as global attractor for all initial conditions. Consequently, whatever the initial state is, after a short transit, each and every closed system approaches it and stays there forever if no other forces applied. However, the notion of intelligence is grounded on the suggestion that logically different states are associated with physically different states and any algorithm is executed as a specific sequence of "jumps" among these states. This consideration poses the major question whether it is possible to execute a given form of intelligence by means of spontaneously executed physico-chemical processes.

Since the second half of 20-th century most promises for resolving this problem have been put on the idea that emergent macroscopic properties spontaneously commence from simple dynamics and/or from spontaneous pattern formation out of simple reaction-diffusion systems. However, the most fundamental inherent contradiction of that idea is that, on the one hand, the emergent behavior and emergent patterns are asymptotically stable and thus they are generically consistent with the idea of thermodynamic equilibrium and one the other hand, they exists only in specific narrow range of control parameters which parametrize the impact of a well-defined steady uniform environment.

Most hopes have been put on the idea that different emergent patterns can be designed so that to operate simultaneously and coherently. However, this idea 
faces both technical and fundamental difficulties. The technical difficulties commence from the fact that different patterns emerge at fine-tuning to different ranges of values of the control parameters which, however, makes most unlikely their simultaneous emergence and simultaneous maintenance. Along with this technical difficulty, another, more crucial one challenges this scenario. It consists of the fact that neither form of intelligence would be adaptable to environmental changes in this framework because the performance of any piece of intelligence would repeat exactly the same. Put the latter in other words, the response of any such system would look more like to a physical law viewed in its traditional setting than to any form of intelligence whose major property is highly non-trivial interplay between uniqueness of any piece of semantics along with its reproducibility viewed as self-sustaining intact the specific causal relations encapsulated in it.

Another non-overwhelming difficulty consists of the fact that any form of self-organization through fine tuning is extremely vulnerable to the tiniest perturbations of the environment.

Outlining, the above considered issues render the idea about appropriate modification of the notion of self-organization so that to make possible the execution of a semantic-like response as a stable spontaneous physical process an ultimate goal.

\section{General Operational Protocol for Execution of Semantic Intelligence by Means of Spontaneous Processes}

One of the major requirements to any form of spontaneously executed intelligence is its stable operation in the course of time and in any environment. That is why I put this requirement in the following setting: namely the setting of un-specified non-uniform ever-changing environment subject to the mild constraint of boundedness alone. The major pay-off of the latter will be that the boundedness of rates and amplitudes alone turns sufficient to provide the substantiation of the decomposition theorem whose exclusive property is to provide grounds for the implementation of semantic intelligence by means of spontaneously executed physico-chemical processes. Thus, the major goal of the present section is to provide delineation of the grounds for the general operational protocol aimed towards sustaining of boundedness of rates and amplitudes, introduced in [1], from the grounds of the point-like approach to response.

Since, as proven by the decomposition theorem, the permanent sustaining of boundedness of rates and amplitudes is sufficient to provide long-term stability of any complex system, let me start with the question how to characterize stability of a system of coexisting homeostatic patterns put in a non-uniform ever-changing environment. It is obvious that no global parameter (e.g. intensity of the impact) is available since different patterns (and their sub-units as well) are put in different local environment; thus, even identical entities respond dif- 
ferently being located at distant spatio-temporal points. This property is generic for all heterogeneous systems and it holds true even for an initially uniform system.

My assertion is that the stability of any system put in a non-uniform ever-changing environment holds true if and only if the interplay of the interactions that proceed in it is able to sustain Euclidean metrics among the distinctive functional properties of all entities and on each and every hierarchical level. Crucial for the substantiation of this assumption is the view on Euclidean metrics as single-size Voronoi tessellation. The latter tacitly presupposes existence of bounded both from below and above ranges of admissible deviations from any given homeostatic pattern so that the pattern properties to stay intact. The bounds of any such range are derived from the requirement that homeostatic patterns on lower hierarchical levels appears as "fine structure" to the higher levels ones. This requirement commences from the necessary condition for sustaining long-term stability of a system: that is the necessity for substantiating a ban over resonances among different patterns. Alongside, keeping an appropriate "distance" from one another renders patterns that come from different hierarchical levels well defined in a long run.

The necessity of keeping both local and global functional metrics Euclidean suggests the idea that the latter is plausible only if the interactions in each and every system are constrained so that to vary within specific margins. The major pay-off is that thus the constraint of boundedness provides holding of the decomposition theorem. Next I will demonstrate that the general operational protocol, introduced in [1], which supervises dynamic processes so that locally accumulated extra energy and matter to dissipate throughout a system serves as grounds for physical implementation of the boundedness of rates and amplitudes. It will be demonstrated also that this protocol operates steadily only when the extra matter and extra energy in every locality does not exceed specific margins imposed so that to provide that corresponding deviations of the local functional metrics stays within the range of the admissible ones.

I start the justification of this idea with the consideration how the notion of a Hamiltonian is to be modified so that to retain the property of being the bearer of identity of the corresponding species in an ever-changing non-specified and non-uniform environment. It is obvious that neither the Hamiltonian nor initial and boundary conditions are steady because the neighborhood of any species which forms it permanently varies put in an ever-changing non-uniform environment. This prompts suggestion that any Hamiltonian and the corresponding initial and boundary conditions vary according to the changes in its neighborhood. Yet, in order to sustain its identity, the variations of the shape of that Hamiltonian must by bounded to be within a specific "strip" "wrapped" around a time-independent steady "skeleton". Initial and boundary conditions are also bounded in specific strips each of which is to be associated with a given state of the time-independent skeleton. Further, in order to sustain the identity of the 
corresponding Hamiltonian, the strips around different states should not overlap. This, however, immediately implies that the changes in initial and boundary conditions and the changes of the corresponding Hamiltonian are correlated. This in turn renders that the changes in the position, momentum, energy and the duration of any transition are also vary bounded within specific margins. In turn, the changes in the current shape of a Hamiltonian and corresponding initial and boundary conditions trigger transitions and so the corresponding species is never in rest. Since this restlessness is generic for any non-steady Hamiltonian, these transitions serve as grounds for implementation of spontaneous processes.

It should be stressed on the high non-triviality of the above assumption. Jumping ahead, the subordination of those variations to the general operational protocol which provides the boundedness of rates and amplitudes renders formation of steady homeostatic patterns of the transitions so that those patterns to appear as emergent ones to the corresponding Hamiltonian's "skeleton"; for example, it includes appearance of extra lines, forbidding certain transitions etc., phenomena well established in condensed matter spectroscopy. Another exclusive property is that it opens the door for a variety of synergetic and antagonistic phenomena whose generic properties is that they are not proportional to the intensity of the corresponding impact.

Before considering the ingredients of the general operational protocol for sustaining boundedness of rates and amplitudes let me remind how the boundedness of rates substantiates the idea for "coordination" of the spontaneous processes which take place at different spatio-temporal points so that to produce long-range correlations among them.

As it has been already established, in order to sustain the identity of species, the changes of all characteristics of any non-steady Hamiltonian due to spontaneous transition must be bounded so that to stay within specific margins. Thus, in order to keep this boundedness permanent, there must be specific constraint imposed on successive transitions. It is worth noting that the constraint imposed on successive transitions substantiates the notion of boundedness of rates. As suggested in Chapter 7 of [1] boundedness of rates is implemented by the stationarity of the corresponding action. A very important property of the proposed stationarity of the action is that it is not associated with any form of extremization and/or optimization. On the contrary, its exclusive property is that it selects those paths where the sequence of transitions does not violate the boundedness of rates. And since, there is no optimization imposed, these paths are generically more than one.

Outlining, two conclusions are of major importance: 1) the first one is that the paths selected by the stationarity of the action provide long-term stable correlations among the transitions which occur on any of those paths. In turn, semantic intelligence can be substantiated in a stable and reproducible way only on those paths; 2) being more than one, the existence of different paths substantiates di- 
versity of semantics.

Let us now remind in details how long-term correlations among events happening at distant spatio-temporal appear due to the boundedness of rates and amplitudes. The importance of that issue arises from the fact that their substantiation justifies the basic premise of the decomposition theorem, namely the premise that the latter is available for bounded, and thus long-term correlated, events. The consideration goes as delineation with the point-like approach to response whose major premise substantiates independence of successive events from one another and thus provides grounds for the availability of the Central Limit Theorem.

I start this consideration with the reminder that the point-like approach to response implies that the latter depends on the current impact and the current state of a system only irrespectively to the previous and future states. To remind that the point-like response gathers its name after the assumption that space and time dimension of the development of any response and/or fluctuation is irrelevant. On the contrary, according to the concept of boundedness, any current response depends explicitly on the current impact and current state but it also implicitly depends on the whole chain of previous states because the realization of any given transition depends on whether the entire chain of all previous ones have been selected from the corresponding subsets of the admissible transitions where the subsets of admissible transitions are selected by the boundedness of rates from the set of all possible ones. In turn, the boundedness of rates produces correlations and inter-dependences among successive events whose generic property is that their memory radius is infinite. In turn, the latter substantiates the fundamental difference with the Markov processes whose hallmark is that the memory radius is always finite. It is worth noting that this difference mathematically substantiates the delineation of the subject of the decomposition theorem (infinite memory radius; hence bounded variables) from the subject of the Central Limit Theorem (finite memory radius; hence independent variables) so that there is no cross-section of both subjects. It is worth reminding that one of the far-going consequences of that delineation is that any form of intelligence is compatible only with the decomposition theorem while the Central Limit Theorem is incapable to provide difference between any sequences of random events from any one which brings any information.

It is worth noting that the replacement of the idea about Markov property of successive responses with the idea of stationarity of the action viewed as an implement for providing boundedness of rates in a long run is free from one of the major controversies of the point-like approach to response discussed in section 2. In turn, its farthest going consequence is that this release renders the grounds for shifting from Central Limit Theorem to the decomposition theorem and thus provides grounds for substantiation for execution of semantic intelligence by means of spontaneous processes.

Further, the stationarity of the action is sustained by a highly non-trivial in- 
terplay among transitions executed on different hierarchical levels because they operate both simultaneously and in sequence but each of them is subject to the constraint of stationarity of the action. In turn, the latter interplay verifies the idea proposed in [1] that the hierarchy of a semantic response is bidirectional, i.e. it operates not only bottom up but top down as well.

It should be stressed that since the stationarity of action is derived from the boundedness of rates, it is obvious that on each and every admissible trajectory it could vary within specific margins set up by it. The stationarity of the action is spontaneously maintained by means of the considered next general protocol which provides that any local accumulation of extra matter and extra energy, which does not exceed specific margins, dissipates throughout the system irrespectively to the chemical nature of the species and their physical properties. In turn, the admissible trajectories associated with each stationary action turns out to be also bounded in a strip "wrapped" around stationary "skeleton". In turn, the fact that the admissible trajectories associated with each and every stationary action are generically more than one enormously expands the range for substantiation of the uniqueness of the semantic response and its diversity.

Outlining, the above suggested idea that the stability of response is substantiated only on paths selected by the permanently imposed constraint of stationarity of the action constitutes the replacement of the controversial notion of point-like approach to response with the idea that in an ever-changing non-uniform environment, the spontaneous processes are executed in a stable way if and only if all changes are kept permanently bounded along the admissible trajectories. In turn, this renders holding of the decomposition theorem which as already has been mentioned provides existence of stable homeostatic patterns.

The next point is to demonstrate that the boundedness of rates and amplitudes and the motion on selected by stationarity of the action trajectories is maintained by means of the existence of a general operational protocol which supervises spontaneously executed dissipation of extra energy and matter accumulated in any given location. It is implemented by putting forward a new general frame for the interactions and wave emission. These frames are introduced in [1] are turns to be ubiquitous because they are insensitive to the chemical nature of the corresponding units and their physical properties and they are insensitive to the details of the simple rules that govern the behavior of those units as well.

Let me start with the ground with the new frame for interactions. I put forward the notion that the interactions are generically non-isolated events and thus their spatial and temporal dimension becomes of key importance. An example of that new notion are the introduced by me in Chapter 3 of [1] non-unitary interactions. They commence as a result of involvement of an extra species in the process of an initially unitary interaction. Since the moment of arrival of the extra species is un-specified, the outcome of the corresponding interaction is different for different moments. It is worth reminding that namely the 
idea that dynamical interactions are isolated from one another serves as grounds for the point-like approach. Its major characteristic is the insignificance of the temporal and the spatial dimension of any isolated interaction for the outcome of its proceeding. I replace the notion about isolation of the dynamical interactions with the notion that dynamical interactions are generically non-isolated. The fundamental difference which this replacement brings about is that temporal and spatial dimension of any interaction become relevant for the outcome because the characteristics of the latter explicitly depend on the moment of intervention of extra species. In turn the cross-section of that interaction becomes a multi-valued function each selection of which corresponds to a given moment of extra-species intervention.

The key implement which provides spontaneous dissipation of any locally accumulated extra-energy is the proposed by me in Chapter 3 of [1] feedback between any non-unitary interaction and appropriate collective modes (i.e. acoustic phonons). Its major property is insensitivity to the chemical nature of the participating species and their physical properties which in turn makes the feedback ubiquitously available for systems of different nature and for every environment. The ubiquity of that feedback is constrained only by the necessity of subordinating its stable operation to the admissible deviations from local functional metrics because the collective modes are well-defined only if the corresponding local deviations from the metrics do not exceed specific margins dictated by the admissible deviations from the metrics. To remind the admissible deviations from the metrics are those which do not compromise the Euclidean metrics viewed as single size Voronoi tessellation. In turn, this provides the operational margins for the feedback between the non-unitary interactions and the collective modes.

The next new general frame considers a new type of waves emitted so that to provide the dissipation of any local accumulation of extra matter. These new waves commence as a new type of solution of non-linear non-autonomous partial differential equations and are mathematically derived by me in Chapter 7 of [1]. The general premises of that derivation are as follows: any local accumulation of extra matter causes a local disturbance of the flow which passes through that spatio-temporal point. The crucial novelty is that this disturbance is parametrized as deviation from the local metrics. This notion is suggested by the very important property of the feedback between the non-unitary interactions and the collective modes which consists of sustaining constant relative velocity among species irrespectively to the chemical nature and the physical properties of participating species [2]. In turn the latter provides a flow to be permanently laminar and sustains the notion of concentration well defined. This consideration justifies the association of any local disturbance of matter balance with a specific local deviation of the diffusion coefficient. In turn, the generic dependence of the diffusion coefficient on deviations of the local concentration provides a new type of solution of the governing reaction-diffusion equation which turns out to be the emission of a matter wave so that its direction is determined 
by the properties of the corresponding gradient of the diffusion coefficient.

It is important to stress on the relation between the conditions for the matter wave emitting and the corresponding metrics. This relation confines the matter wave emitting to be available only when the corresponding deviations from the local metrics belong to the admissible deviations from the corresponding functional metrics. To remind that the feedback between non-unitary interactions and collective modes which provides dissipation of any locally accumulated extra-energy is also constrained by the margins of the admissible deviations from the functional metrics.

Outlining, the operational margins of the general protocol for spontaneous dissipation of locally accumulated extra-energy and extra-matter are explicitly related to the admissible deviations from the corresponding functional metrics. In turn, this implements an explicit relation of the notion of boundedness of rates and amplitudes with the long-term stability of the corresponding system.

Summarizing, I replace the notion of point-like approach to response with the notion about existence of general operational protocol which supervises dynamics so that to sustain permanently boundedness of rates and amplitudes by means of spontaneously executed dissipation of extra-matter and extra-energy in any given locality. The ubiquity of this protocol is controlled by the general condition for subordination of the extra-energy and extra-matter amount to match the admissible deviations from the local functional metrics. Further, the substantiation of that protocol is grounded on the notion about non-isolated interactions and matter wave emitting where the latter is viewed as a new type of solution of non-linear non-autonomous partial differential equations. These novel notions render the spatial and temporal dimension of any initially unitary interaction of key importance which culminates in the exclusive property of the corresponding cross-section to become a multi-valued function. As a result, the response of any complex system is executed as spontaneous non-stop jumps between successive homeostatic patterns. This result is fundamentally different from the notion of thermodynamic equilibrium viewed as a global attractor for all initial conditions which notion is central for the point-like approach to response. Put it in other words, I replace the notion of thermodynamic equilibrium viewed as a global attractor with the notion of stability implemented by a general operational protocol for providing boundedness of rates and amplitudes through spontaneous dissipation of locally accumulated extra-matter and extra energy if only the latter is bounded within specific margins set by the admissible deviations from local functional metrics.

\section{Implementation of Causal Relations: New Approach to the Notion of Self-Organization}

The major idea put forward in the previous section is that there exists general operational protocol which supervises the interactions so that make possible keeping permanent boundedness of rates and amplitudes on selected trajectories 
where the action is stationary. In turn, the sustaining of boundedness of rates and amplitudes onto certain trajectories provides holding of the decomposition theorem which in turn provides existence of specific steady homeostatic patterns for each and every location where these trajectories passes through. The major property of any of these patterns is that its properties do not exactly match the properties of the corresponding structure. In turn, the lack of one-to one correspondence between the properties of, for example, the corresponding Hamiltonian's "skeleton" and the emergent functional properties could give rise to, appearance of extra lines, could forbid certain transitions etc., phenomena well established in condensed matter spectroscopy. Another exclusive property is that it opens the door for a variety of synergetic and antagonistic phenomena whose generic properties is that they are not proportional to the intensity of the corresponding impact.

Other generic property of the homeostatic patterns is that they may be different on different trajectories. This property is exclusive for the setting of boundedness and has no analog at the current science where the requirement about optimization selects a single match between structural and functional properties.

Further, the boundedness of action, viewed as implement for permanent sustaining of the boundedness of rates, also renders existence of specific homeostatic patterns associated with each trajectory. In turn this provides "fine structure" of the diversity of semantics. Thus, the existence of a "fine structure" specific for each trajectory, enormously increase the range of diversity of semantics by means of setting up the grounds for "hierarchy" of that diversity. Alongside, it enormously expands the range for substantiation of the uniqueness of the semantic response.

Thus, the ubiquitous existence of homeostatic patterns whose major property is insensitivity to the details of environmental fluctuations opens the door to a new form of causality viewed as emergent steady functionality. Thus, in [1] I have introduced the notion of the meaning of a semantic unit as the performance of a non-mechanical engine built on the corresponding trajectory for the purpose to make the corresponding causality irreducible to the set of the binary relations among the constituents and to view it as an emergent property. This comes on the contrary to the traditional algorithmic theory where causality is viewed as reducible to steady binary relations among successive states of hardware only. The major weak point of the current view is two-fold: 1) the reduction of causality to binary relations implies lack of continuity in causality which in turn renders truly causal relations indistinguishable from a random sequence; 2) being steady relations until an external intervention takes place, there is no route for spontaneous execution of any sequence of causal relations.

The greatest advantage of the withdrawal from the reducibility of causality to steady binary correlations is provided by the exclusive property of even the simplest form of causality viewed as emergent steady pattern that is to be covariant with respect to time-translational invariance and independent from the refer- 
ence frame chosen for its description. This is an exclusive property provided by the decomposition theorem according to which, any homeostatic pattern viewed as a piece of causality, is additively superimposed onto a noise component so that both the shape of the latter and structure of the former turn out insensitive to the details of the current environment. Consequently, the homeostatic pattern is insensitive to positions on the arrow of time of both the initial and the final moments of its execution. The same insensitivity holds for the specific location of any homeostatic pattern in the space. In turn, the insensitivity of the properties of any homeostatic pattern to its current spatio-temporal position justifies the covariance of the causality encapsulated in each and every homeostatic pattern.

Thus, the decomposition theorem enormously expands the grounds for time translational invariance of causality and its independence from the reference frame rendering them available for complex systems put in a non-uniform ever-changing environment. To compare, the point-like approach to response provide these properties for steady uniform environment only.

It is worth noting that the above established covariance of causality is an exclusive property of the self-organized functionality only. A crucially important consequence consists of the fact that the latter allows it's commence from structures of different spatio-temporal metrics. Note however, that while the spatio-temporal metrics could be any (Euclidean, Riemann etc.), the metrics of any stable functional organization is always Euclidean. In turn, this once again justifies the idea proposed in [1] that the functionality is an emergent phenomenon which commences from highly non-trivial specific interplay among many different interactions supervised by the above discussed general operational protocol for sustaining boundedness.

The fact that the functionality signifies an emergent phenomenon makes possible to put forward a novel prospective on self-organization: it is substantiated by means of spontaneous motion among a hierarchy of steady homeostatic patterns. The ultimate goal of this motion is to "adjust" the response of a system, viewed as specific self-organized functionality, so that to provide its long-term stability in an ever-changing non-uniform environment.

There are three exclusive properties of thus modified notion of self-organization:

1) The emergent behavior is characterized by the fact that, although the properties of any homeostatic pattern are derived from the structure, the generic lack of optimization renders no single match and no one-to-one correspondence between structure and functionality. Put it in other words, the functionality is "emergent" with respect to the structure. In turn, this justifies the view put forward in [1] statement that the most effective semantics must not to be associated every time with the fastest computers and/or those ones whose maintenance requires least losses.

2) A great advantage of this type of self-organization is that it operates steadily at any concrete specification of ever-changing non-uniform environment let 
alone the latter is bounded. Thus, semantics is not constrained to the narrow range of appropriate values of the corresponding control parameters selected by the process of fine-tuning as the present-day views on it prescribe.

3) The hierarchy of response is bi-directional which means that it goes both bottom up and top down. Its exclusive property is that both directions are entangled in a highly non-trivial interplay because their simultaneous operation makes them supervise each other.

\section{Conclusions}

The major goal of the present paper is to consider an adequate modification of the current idea of self-organization so that to put grounds for execution of certain new form of intelligence through spontaneous physico-chemical processes. It has been demonstrated that this idea challenges the very fundament of the current interdisciplinary science. A systematic overcoming of those difficulties is achieved in the setting of the recently proposed by the author concept of boundedness [1].

The focus in the present paper is to establish the physical grounds for a novel concept of self-organization. It is demonstrated that: 1) the basics of the point-like approach to response, widely adopted in the current science, are inherently contradictive and are unable to provide spontaneous execution of any form of intelligence; 2 ) it is demonstrated that there exists general operational protocol supervising the dynamical behavior of any complex system which turns sufficient to provide the execution of semantic intelligence by means of spontaneous physico-chemical processes in an unspecified ever-changing non-uniform environment let alone the latter is bounded.

The greatest advantage of the novel notion of self-organization is the covariance of causality encapsulated in any piece of semantics because it is provided with a great diversity of individuality viewed as the properties of the current response and reproducibility viewed as causality encapsulated in any of the homeostatic patterns which the current non-mechanical engine is built on. Alongside, the consistency of the functional metrics, which is always Euclidean, with any metrics of the space-time renders the proposed in the present paper notion of self-organization ubiquitously available. It is now available for systems ranging from nano-objects, living organisms to cosmological ones.

\section{Conflicts of Interest}

The author declares no conflicts of interest regarding the publication of this paper.

\section{References}

[1] Koleva, M.K. (2012) Boundedeness and Self-Organized Semantics: Theory and Applications. IGI-Global, Hershey, PA.

[2] Koleva, M.K. (2015) Journal of Modern Physics, 6, 1149-1155. https://doi.org/10.4236/jmp.2015.68118 